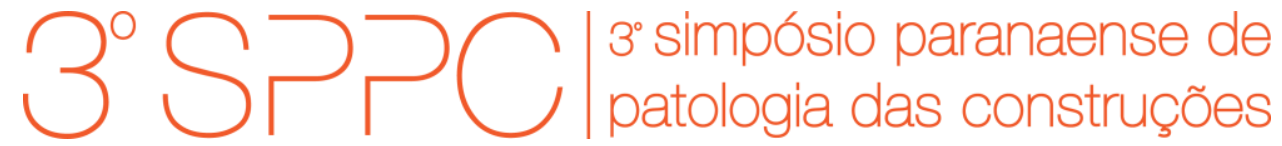

ISSN 2526-7248 artigo n. 3SPPC1009, pp. 96-105, 2018

\title{
Resistividade elétrica superficial do concreto com cimento CP V ARI: experimental $x$ calculado
}

\author{
Kleina, Mário Sergio Mandú 1; Müller, Rodrigo Oliveira 2; Volert, Ricardo Cezar ${ }^{3}$; \\ Medeiros-Junior, Ronaldo A. ${ }^{4}$ \\ ${ }^{1}$ Mestrando, Universidade Federal do Paraná, mario.kleina@lactec.org.br \\ Engenheiro civil, Universidade Tuiuti do Paraná, eng.rodrigomuller@gmail.com \\ ${ }^{3}$ Graduando em Física, Universidade Tecnológica Federal do Paraná, \\ ricardovolert@gmail.com \\ ${ }^{4}$ Professor, Universidade Federal do Paraná, medeirosjunior.ufpr@gmail.com
}

Resumo: O ensaio de Resistencia Elétrica Superficial (RES) é um importante parâmetro para medir a durabilidade e vida útil de estruturas de concreto, pois fornece parâmetros para mensurar a permeabilidade do concreto, os possíveis ataques de agentes agressivos, sendo ainda um ensaio não-destrutivo. Este estudo trata da comparação do ensaio de RES entre quatro diferentes traços de concreto utilizando cimento Portland CP V ARI, variando o consumo de cimento entre 250 e $400 \mathrm{~kg} / \mathrm{m}^{3}$. Os resultados experimentais de resistividade foram obtidos com o Método dos Quatro Pontos, também chamado de Método de Wenner, aos 7 e 28 dias de idade, em corpos de prova cilíndricos de $\$ 10 \times 20 \mathrm{~cm}$. Tais resultados foram comparados à resistividade do concreto calculada através do modelo proposto por Andrade e D'Andréa (2011) [1], na qual induzem uma metologia para a determinação da RES de um concreto baseado na lei de Archie e Powers, que se mostrou valido e coerente.

Palavras-chave: Resistividade Elétrica Superficial, Método de Wenner, Lei de Archie e Powers.

Abstract: Electrical resistivity test is an important parameter for measuring the durability and service life of concrete structures, since it provides parameters to measure the permeability of concrete and possible attacks of aggressive agents. This study deals with the comparison of electrical resistivity test with four different concrete proportions using ordinary Portland cement (CP V ARI), varying the cement content between 250 and $400 \mathrm{~kg} / \mathrm{m}^{3}$. The experimental results of resistivity were obtained by the Four-Electrode Method (Wenner's Method), at 7 and 28 days of age, in $\phi 10 \times 20 \mathrm{~cm}$ cylindrical specimens. These results were compared to the concrete resistivity calculated through the model proposed by Andrade and D'Andréa (2011) [1], in which they induce a methodology for the determination of electrical resistivity of a concrete based on the Archie and Powers models.

Keywords: Electrical resistivity, Wenner Method, Archie and Powers model. 


\section{Introdução}

É de notório conhecimento que o concreto armado é um dos materiais mais utilizados do planeta, seja por sua facilidade de utilização, moldagem das mais variadas formas ou custo relativamente baixo. Atualmente existem vários ensaios para testar suas qualidades e ter controle de aceitação. Neste contexto, pode-se citar a resistividade elétrica do concreto, que pode ser entendido como a capacidade do concreto em resistir ao fluxo de corrente elétrica de natureza iônica na solução aquosa contida na rede de poros comunicantes (bolhas, interstícios, canais capilares). Esta propriedade é um importante parâmetro para determinar a vida útil da estrutura, podendo fornecer informações sobre a microestrutura do concreto, porosidade e o conteúdo de água nas primeiras idades [1].

A água no interior do concreto pode carregar vários agentes agressivos, como os íons de cloreto. Segundo Glass [2], a resistividade é afetada pela presença de íons cloreto na solução aquosa e pela temperatura. A total saturação do concreto é a condição ideal para o fluxo de íons. O fluxo ocorre por meio de filme de água absorvida na superfície das paredes dos poros. Todavia, quando o concreto está saturado ele comporta-se como um material semicondutor com valores de resistividade elétrica da ordem de $10^{2} \Omega \mathrm{m}$, enquanto que seco é considerado um material isolante elétrico, com resistividade da ordem de $10^{6}$ $\Omega m$ [3]. Uma das facilidades para o uso da resistividade do concreto está no fato deste ser um ensaio não destrutivo, permitindo sua realização contínua em uma única peça ao longo do tempo.

A resistividade elétrica do concreto é uma propriedade que depende principalmente da composição química da solução aquosa dos poros, da estrutura dos poros, da umidade e da temperatura do concreto [4]. Fatores que influenciam esta propriedade são:

- Relação água/cimento: A relação água/cimento influencia consideravelmente a estrutura porosa da pasta de cimento, especificamente no volume total de poros e na quantidade de poros de maiores diâmetros. Quanto menor é a relação água/cimento, menor será o volume de poros e a probabilidade de existirem poros com maiores diâmetros e interconectados [5];

- Cura e grau de hidratação do cimento: De acordo com Neville [6], em concretos convencionais, a cura tende a garantir o mais alto grau de hidratação possível, possibilitando obter maior resistência e menor permeabilidade, além de minimizar os efeitos da retração autógena. Em concretos de alto desempenho, a cura é ainda mais importante, devido à baixa velocidade de exsudação no estado plástico e auto secagem e gradientes térmicos no concreto endurecido.

Helene [3] afirma que a resistividade elétrica do concreto depende do grau de hidratação do cimento. Schulte et al. [7] discutem que a variação da resistividade elétrica do concreto em condições semi saturadas ocorre devido a condução iônica através da camada de água adsorvida 
nas paredes dos poros, enquanto nos concretos muito secos, a condutividade diminui, e o concreto atua como isolante elétrico. O grau de saturação na rede de poros capilares do concreto pode variar em função das condições atmosféricas do ambiente no qual esteja exposto. As chuvas e a umidade relativa alteram o conteúdo de umidade do concreto [8]. Whitington et al. [9] afirmam que a condutividade elétrica do concreto é função do tempo (ou idade do concreto).

Woelf e Lauer [10], comparando corpos de prova curados ao ar com corpos de prova submetidos à cura úmida, observaram que houve um aumento significativo da resistividade elétrica aos 23 dias nos corpos de prova curados ao ar. Esse aumento é devido à evaporação da água livre, que é condutora de corrente pelo concreto;

- Agregados: Whiting e Nagi [11] asseguram que a resistividade elétrica do agregado é muitas vezes maior que a da pasta de cimento.

Segundo Monfore (1968 apud Santos [5]), concretos com agregados de origem calcária e arenítica apresentam uma resistividade elétrica muito menor que concretos elaborados com granito.

Os agregados normalmente têm resistividades maiores que a pasta do cimento, o que faz que a maior parte da corrente elétrica seja conduzida através da pasta [12];

- Temperatura: O aumento da umidade e da temperatura resulta em diminuição da resistividade elétrica $[3,6]$.

Whiting e Nagi [11] constataram que a resistividade do concreto depende da temperatura. A temperatura tem influência sobre a solução presente nos poros (eletrólito). Aumentando a temperatura, a viscosidade da solução é diminuida e a mobilidade iônica tende a aumentar.

Castellote et al. [8] afirmam que a resistividade elétrica, sendo o inverso da condutividade, depende da temperatura. Um aumento da temperatura comumente resulta em diminuição da resistividade devido ao aumento da mobilidade e interação iônicas. Esses autores sugerem a adoção de $25^{\circ} \mathrm{C}$ como uma temperatura de referência para ensaios.

- Carbonatação: A carbonatação gera a precipitação de carbonato de cálcio nos poros, o que provoca o endurecimento da superfície do concreto, havendo um aumento significativo da resistividade elétrica na superfície [13].

- Adições minerais: Segundo Santos [5], as adições minerais, tais como cinza volante, sílica ativa e escória de alto-forno provocam o refinamento dos poros da matriz, por ação química e/ou física, e diminuem a concentração iônica da solução. Sendo assim, o concreto com adições minerais apresenta uma maior resistividade elétrica quando em comparação com um concreto convencional. A densificação da matriz e 
o aumento da quantidade de poros de menores diâmetros dificultam a condução da corrente através do concreto e, portanto, aumentam a resistividade elétrica.

- Teor de umidade. A umidade relativa é um parâmetro decisivo para o grau de saturação dos poros, que por sua vez controla a resistividade elétrica do concreto, principalmente na região superficial. Segundo Helene [3], a evaporação que é um fenômeno de superfície, ocorre muito mais rapidamente que o transporte de água e secagem interna do concreto.

Existem vários métodos experimentais para determinação da resistividade elétrica do concreto, como o Método da Resistividade Elétrica Volumétrica; Método dos Dois Eletrodos; Método do Eletrodo Externo; e o Método dos Quatro Pontos (Método do Wenner, escolhido para aplicação neste estudo).

Apesar de ser fácil e de simples aplicação, algumas medidas de precaução devem ser realizadas para aplicação do método de Wenner. Segundo Hoppe [4], fatores como geometria dos corpos de prova, superfície de contato entre os eletrodos e o concreto, heterogeneidade do concreto, presença de armaduras, tipo de onda, frequência e intensidade da corrente aplicada devem ser cuidadosamente considerados durante o teste.

Neste contexto, Andrade e D'Andréa [1] propuseram uma metodologia capaz de estimar e dosar o concreto a partir de um valor de RES pré-estabelecido medido aos 28 dias. Esta metodologia é um avanço no modelo de Andrade [14], baseado também na lei de Archie e Powers (Eq.1).

$$
\rho=a \cdot \rho_{o} \cdot \mathcal{E}^{-T}
$$

Onde:

- $\rho$ é a RES esperada para o concreto ( $\Omega . m)$;

- a é uma constante, aproximadamente igual a 1 [15];

- $\varepsilon$ é a porosidade do concreto, que depende basicamente da relação a/c;

- T é um parâmetro relacionado com a conectividade e tortuosidade dos poros do concreto - entre 1 e 2, de acordo com Wong et al. [18] - que pode ser estimado experimentalmente por ajuste de dados. Para o caso do cimento CP V ARI, o coeficiente recomendado é de 1,7;

- $\rho_{o}$ é a resistividade elétrica da solução nos poros do concreto. Este parâmetro é de difícil mensuração. Portanto, Goñi e Andrade [19] recomendam o uso do valor fixo de $0,5 \Omega$.m, pois se o concreto permanecer em solução alcalina, a diferença é muito pequena, não sendo representativa.

A metodologia proposta (Eq. 1) possibilita atestar se o valor de RES cumpre a demanda do projetista mediante a determinação experimental da RES do concreto saturado em água aos 28 dias. Trata-se de um método interativo, 
onde o ciclo é finalizado quando o valor de RES obtido é igual ou maior ao valor de RES requisitado pelo projetista.

O objetivo deste estudo é comparar os valores experimentais de RES com os valores estimados a partir das leis de Archie e Powers.

\section{Materias e Métodos}

De modo a testar a aplicação da Eq. (1), foram definidos diferentes traços de concretos. Posteriormente, a RES esperada baseada na lei de Archie e Powers foi calculada. Esses resultados foram comparados aos valores obtidos experimentalmente, através da moldagem de corpos de prova de concreto.

Os concretos estudados foram dosados utilizando quatro traços diferentes, variando consumo de cimento e a relação água/cimento de modo a manter o abatimento do tronco cone em $100 \pm 10 \mathrm{~mm}$, conforme apresentado na Tabela 1. Os insumos utilizados foram o cimento Portland CP V ARI e agregado basáltico, britado em granulometrias de areia e brita.

Tabela 1: Quantidade de material para $1 \mathrm{~m}^{3}$ de concreto

\begin{tabular}{l|llll}
\hline & \multicolumn{4}{|l}{ Massa (kg) } \\
& E-3432 & E-3433 & E-3434 & E-3435 \\
\hline Materiais & 400 & 347 & 290 & 250 \\
\hline Cimento CP V ARI & 742 & 877 & 934 & 950 \\
\hline Areia de britagem produzida em laboratório & 1076 & 1084 & 1090 & 1077 \\
\hline Brita produzida em laboratório & 4,4 & 3 & 2,3 & 1,8 \\
\hline Aditivo plastificante & 0,41 & 0,51 & 0,66 & 0,88 \\
\hline Relação a/c & 110 & 115 & 110 & 105 \\
\hline Abatimento (slump) & & & & \\
\hline
\end{tabular}

Para cada traço de concreto, foram confeccionados quatro corpos de prova cilíndricos $\phi 100 \times 200 \mathrm{~mm}$, moldados em uma única camada por meio de vibrador mecânico (Figura 1), de acordo com NBR 5738 [16]. Após a moldagem, realizou-se a cura das amostras com a manutenção de um filme de PVC por $24 \mathrm{~h}$. Posteriormente, os corpos de prova foram armazenados em câmara úmida até atingir as idades de ensaio.

Também foi avaliada a evolução da resistência mecânica dos concretos para as idades de 7 e 28 dias. Os ensaios foram realizados segundo as diretrizes da NBR 5739 [17], utilizando uma prensa marca EMIC, modelo PC 200, com capacidade para $200 \mathrm{tf}$, devidamente calibrada. 


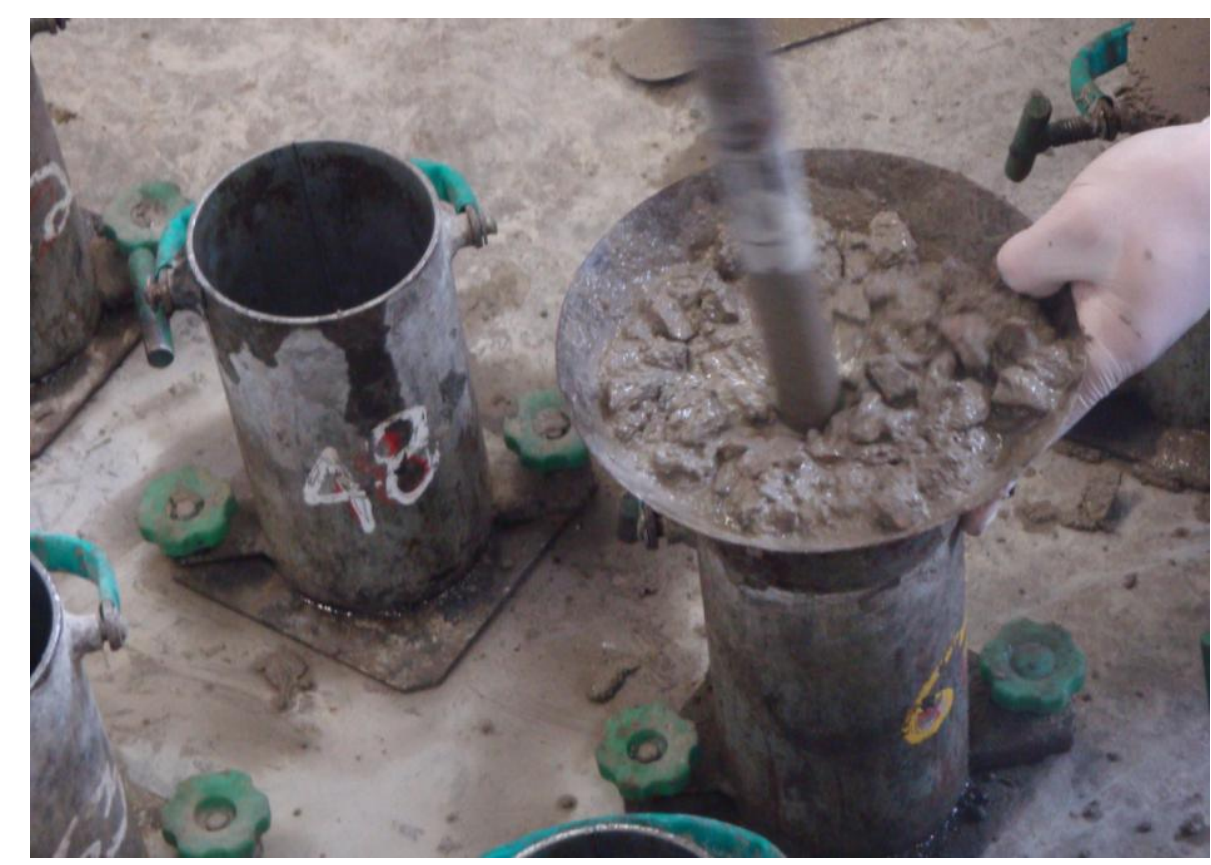

Figura 1: Moldagem dos corpos de prova

Para a determinação da resistividade elétrica do concreto, foi utilizado o equipamento Resipod, conforme ilustrado na Figura 2, com espaçamento entre eletrodos de $50 \mathrm{~mm}$.

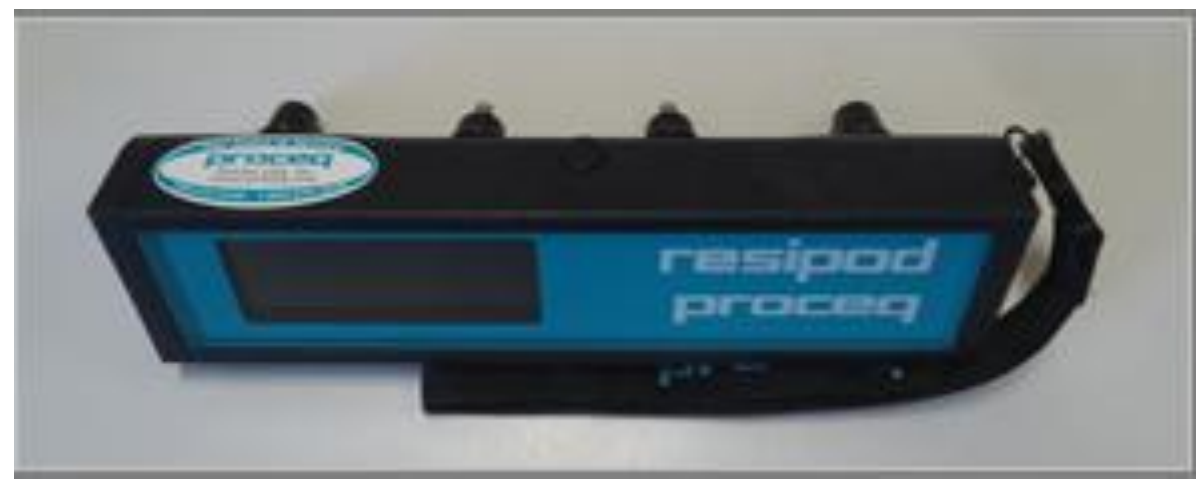

Figura 2: Equipamento para medição da RES

\section{Resultados e Discussões}

Os resultados dos parâmetros de resistividade calculados, de acordo com o modelo proposto por Andrade e D'Andréa [1], para cada um dos traços de concreto com idade de 28 dias estão apresentados na Tabela 2.

A Tabela 3 apresenta os valores de resistividade obtidos após a medição com o Resipod. O valor representa a média de três determinações para cada um dos corpos de prova analisados na idade de 28 dias. Os valores estão colocados juntamente com os valores calculados de modo a se estabelecer um comparativo.

Observa-se que a resistividade é maior para os traços com menor relação água/cimento. Quando comparados os valores calculados e medidos 
experimentalmente, verifica-se a proximidade dos mesmos, sendo que os valores calculados estão a favor da segurança para todos os casos analisados. Os traços extremos apresentaram uma maior variação entre os valores obtidos e calculados, corroborando para a limitação do método com relação ao consumo de cimento.

Tabela 2: Parâmetros calculados através do método proposto por Andrade e D’Andréa [1]

\begin{tabular}{l|llll}
\hline \multicolumn{1}{c|}{ Parâmetros calculados } & E-3432 & E-3433 & E-3434 & E-3435 \\
\hline Relação água/cimento & 0,41 & 0,51 & 0,66 & 0,88 \\
\hline Grau de hidratação do cimento $(\alpha)$ & 0,70 & 0,75 & 0,80 & 0,84 \\
\hline Porosidade da pasta de cimento hidratada $\left(\varepsilon_{\mathrm{p}}\right)$ & 0,21 & 0,29 & 0,38 & 0,48 \\
\hline Volume de pasta no concreto $(\gamma)$ & 0,40 & 0,32 & 0,28 & 0,27 \\
\hline Porosidade do concreto $(\varepsilon)$ & 0,09 & 0,09 & 0,11 & 0,13 \\
\hline Resistividade do concreto $(\rho)$ em $\Omega \cdot m$ & 32,3 & 28,1 & 22,1 & 15,8 \\
\hline
\end{tabular}

Tabela 3: Resistividade obtida pela medição com o Resipod

\begin{tabular}{lllll}
\hline & E-3432 & E-3433 & E-3434 & E-3435 \\
\hline Resistividade calculada $(\Omega . m)$ & 32,3 & 28,1 & 22,1 & 15,8 \\
\hline Resistividade obtida em ( $\Omega$.m) & $\mathbf{3 7 , 8}$ & $\mathbf{2 8 , 3}$ & $\mathbf{2 4 , 2}$ & $\mathbf{1 9 , 8}$ \\
\hline Relação obtida/calculada & 1,17 & 1,01 & 1,10 & 1,26 \\
\hline
\end{tabular}

A Figura 3 apresenta a evolução dos valores de resistência à compressão e RES dos traços analisados ao longo do tempo.

O aumento da resistência mecânica é coerente tanto para a diminuição da relação água/cimento, quanto para o aumento da idade. A resistência à compressão inicial (7 dias) foi de aproximadamente $85 \%$ da resistência aos 28 dias para todos os traços analisados, corroborando para o fato da utilização do cimento de alta resistência inicial (CP V ARI) nas dosagens. Com relação à resistividade, verificou-se um aumento mais significativo ao longo do tempo (cerca de 60 a 80\%). Tal fato justifica a limitação do método para a idade de controle de 28 dias. 


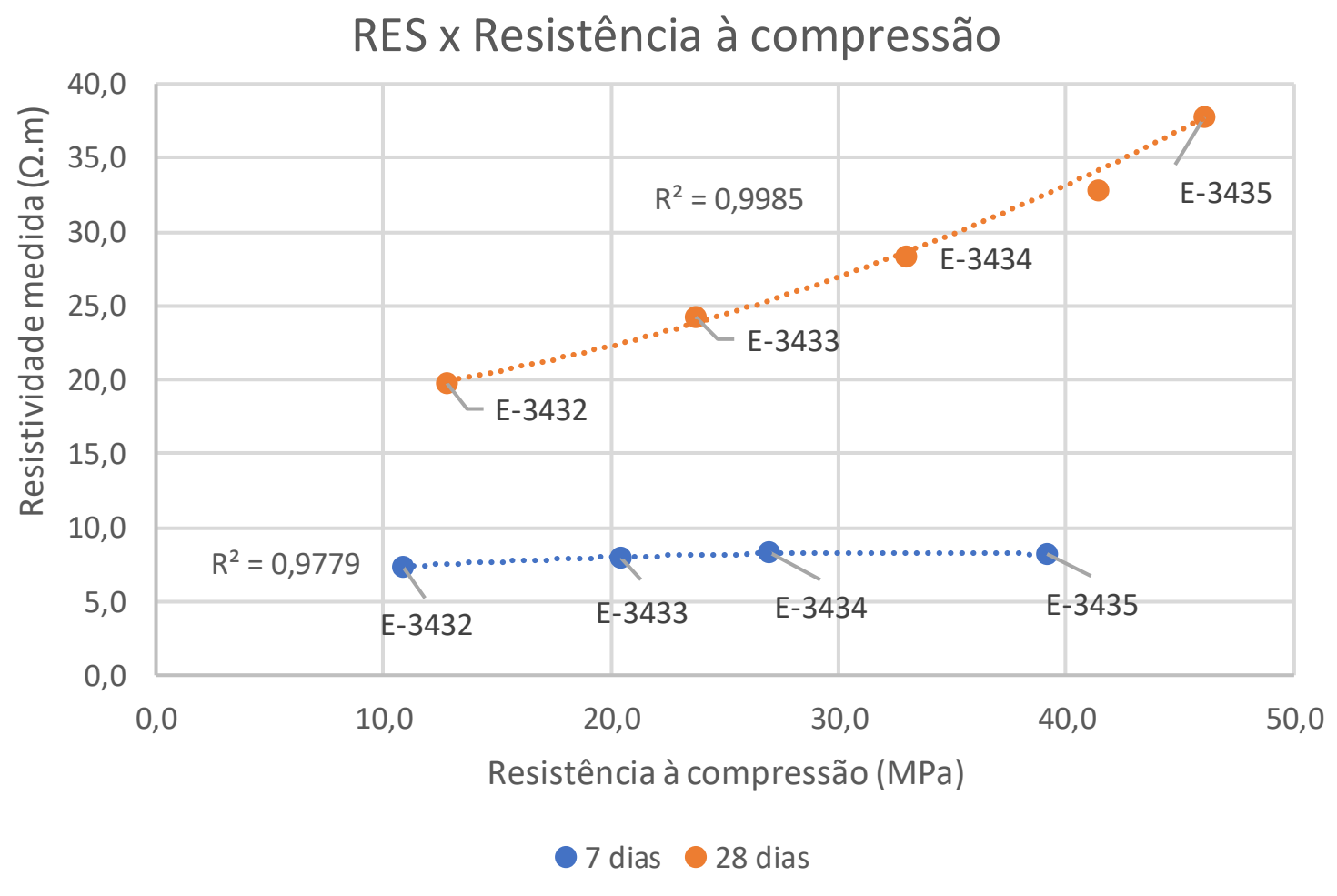

Figura 3: Comparativo entre a RES medida e a resistência à compressão dos concretos

\section{Considerações Finais}

O método de estimativa da resistividade elétrica após 28 dias de cura úmida se apresentou coerente. A validação através da escolha de traços de concreto utilizando cimento CP V ARI com diferentes consumos e relações a/c demonstrou considerável precisão, com valores obtidos sempre a favor da segurança.

\section{Referências:}

[1] ANDRADE, C.; D'ANDRÉA, R. La resistividad eletrica como parâmetro de control del hormigón y de su durabilidad. Revista ALCONPAT, v.1, n. 2, p. 93-101, 2011.

[2] GLASS, G. K. Et al. Factors Affecting Steel Corrosion In Carbonated Mortars. Corrosion Science, v.32. 1991.

[3] HELENE, P. R. Contribuição ao estudo da corrosão em armaduras de concreto armado. Tese (Livre-Docência) - Escola Politécnica da Universidade de São Paulo, Departamento de Engenharia Civil, São Paulo, 1993.

[4] HOPPE, T. Resistividade elétrica do concreto contendo diferentes teores de cinza de casca de arroz. Dissertação (Mestrado) - Universidade Federal de Santa Maria, Santa Maria, 2005. 
[5] SANTOS, L. Avaliação da resistividade elétrica do concreto como parâmetro para a previsão da iniciação da corrosão induzida por cloretos em estruturas de concreto. Dissertação (Mestrado em estruturas e Engenharia Civil) - Universidade de Brasília, Brasília, 2006.

[6] NEVILLE, A. M. Propriedades do concreto. São Paulo. PINI. 1997.

[7] SCHULTE, C.; MADER, H.;WITTMANN, F.H. "Electric Conductivity of Hardened Cement Paste at Different Moisture Contents". Cement and Concrete Research 8, Nr. 3, S, 1978. pp.359-368.

[8] CASTEllOTE, M.; ANDRADE, C.; ALONSO, M. C. Standardization, to a reference of $25^{\circ} \mathrm{C}$, of electrical resistivity for mortars and concretes in saturated or isolated conditions. ACI Materials Journal, v.99, No.2, p.385390, 2002.

[9] WHITTINGTON, H. W; MCCARTER, W. J; FORDE, M. C. The conduction of electricity through concrete. Magazine of Concrete Research, Vol 33, n. 114, 1981. p. 48-60.

[10] WOELF, G. A.: LAUER, K. The Electrical Resistivity of Concrete With Emphasis on the use of electrical resistance for measuring moisture content. Cement, Concrete and Aggregares, v. 1, n.2, 1979.

[11] WHITING, D. A.; NAGI, M. A. Electrical resistivity of concrete - A literature review. 2003. 57p. R\&D Serial n. 2457. Portland Cement Association. Skokie, Illnois, EUA.

[12] LENCIONI, J. W. Estudos sobre resistividade elétrica superficial em concreto: análise e quantificação de parâmetros intervenientes nos ensaios Tese (Doutorado em Engenharia de Infraestrutura Aeronáutica) -Instituto Tecnológico de Aeronáutica, ITA, Brasil, 2011.

[13] MILLARD, S. G. Reinforced concrete resistivity measurement techniques. Proceedings of InstitLute if Civil Engineers. Vol. 91, parte 2, (1991), p. 7188.

[14] ANDRADE, C. Calculation of initiation and propagation periods of servicelife of reinforcements by using the electrical resistivity. International Symposium on Advances in Concrete through Science and Engineering, RILEM Symposium, Evanston, 2004.

[15] GARBOCZI, E.J. Permeability, and microstructural parameters: A critical review. Cement and Concrete Research, v.20, p.591-601, 1990.

[16] NBR 5738 (2016). Cimenti Portland de Alta Resistência inicial. Associação Brasileira de Normas Técnicas - ABNT, Rio de Janeiro, Brasil.

[17] NBR 5739 (2007). Concreto - Ensaio de compressão de corpos-de-prova cilíndricos. Associação Brasileira de Normas Técnicas - ABNT, Rio de Janeiro, Brasil.

[18] WONG, P.; KOPLIK, J.; TOMANIC, J. P. Conductivity and permeability of rocks. Physical Review, B30, 6606, 1984. 
KLEINA, M. S. M.; MÜLLER, R. O.; MEDEIROS-JÚNIOR, R. A., RESISTIVIDADE ELÉTRICA SUPERFICIAL DO CONCRETO COM CIMENTO CP V ARI: EXPERIMENTAL X CALCULADO. $3^{\circ}$ Simpósio Paranaense de Patologia das Construções (30 SPPC), artigo 3SPPC1009, pp. 96 - 105, 2018. DOI: 10.4322/2526-7248.009

[19] GOÑI, S.; ANDRADE, C. Synthetic concrete pore solution chemistry and rebar corrosion rate in the presence of chlorides. Cement and Concrete Research, v.20, p. 525-539, 1990. 